Published in final edited form as:

Gastroenterology. 2015 June ; 148(7): 1353-1361.e3. doi:10.1053/j.gastro.2015.02.050.

\title{
Population-representative Incidence of Drug-induced Acute Liver Failure Based on an Analysis of an Integrated Healthcare System
}

\author{
David S. Goldberg, MD, MSCE ${ }^{1,2,3,4}$, Kimberly A. Forde, MD, MHS ${ }^{1,2,3}$, Dena M. Carbonari, \\ MS $^{2,3}$, James D. Lewis, MD, MSCE ${ }^{1,2,3,4}$, Kimberly B. F. Leidl, MPH ${ }^{2}$, K. Rajender Reddy, \\ MD1, Kevin Haynes, PharmD, MSCE ${ }^{2,3,5}$, Jason Roy, PhD $^{2,3}$, Daohang Sha, PhD $^{2}$, Amy R. \\ Marks, MPH ${ }^{6}$, Jennifer L. Schneider, MPH ${ }^{6}$, Brian L. Strom, MD, MPH ${ }^{2,3,7}$, Douglas A. \\ Corley, MD, PhD ${ }^{6}$, and Vincent Lo Re III, MD, MSCE $2,3,8$ \\ ${ }^{1}$ Division of Gastroenterology, Department of Medicine, Perelman School of Medicine, University \\ of Pennsylvania, Philadelphia, PA, USA \\ ${ }^{2}$ Center for Clinical Epidemiology and Biostatistics, Department of Biostatistics and Epidemiology, \\ Perelman School of Medicine, University of Pennsylvania, Philadelphia, PA, USA \\ ${ }^{3}$ Center for Pharmacoepidemiology Research and Training, Perelman School of Medicine, \\ University of Pennsylvania, Philadelphia, PA, USA
}

\footnotetext{
(C) 2015 Published by The AGA Institute

Corresponding Author and Contact for Reprint Requests: David Goldberg, MD, MSCE, Center for Clinical Epidemiology and Biostatistics, 730 Blockley Hall, 423 Guardian Drive, Philadelphia, PA 19104, Phone: 215-746-8598, Fax: 215-349-5915, david.goldberg@uphs.upenn.edu.

Disclosures: None of the authors have any conflicts of interest that are relevant to the manuscript.

Author Contributions

David Goldberg: Study concept and design, case adjudication, analysis and interpretation of data, drafting of the manuscript, statistical analysis.

Kimberly Forde: Study concept and design, case adjudication, critical revision of the manuscript for important intellectual content Dena Carbonari: Study concept and design, analysis and interpretation of data, drafting of the manuscript, critical revision of the manuscript for important intellectual content.

James Lewis: Study concept and design, analysis and interpretation of data, critical revision of the manuscript for important intellectual content.

Kimberly Leidl: Study concept and design, acquisition of data.

K. Rajender Reddy: Study concept and design, case adjudication, critical revision of the manuscript for important intellectual content. Kevin Haynes: Study concept and design, analysis and interpretation of data, acquisition of data.

Jason Roy: Study concept and design, analysis and interpretation of data, critical revision of the manuscript for important intellectual content.

Daohang Sha: Study concept and design, analysis and interpretation of data, critical revision of the manuscript for important intellectual content.

Amy Marks: Study concept and design, analysis and interpretation of data, acquisition of data.

Jennifer Schneider: Study concept and design, analysis and interpretation of data, acquisition of data.

Brian Strom: Study concept and design, analysis and interpretation of data, critical revision of the manuscript for important intellectual content.

Douglas Corley: Study concept and design, analysis and interpretation of data, drafting of the manuscript, critical revision of the manuscript for important intellectual content.

Vincent Lo Re III: Study concept and design, analysis and interpretation of data, drafting of the manuscript, critical revision of the manuscript for important intellectual content.

Publisher's Disclaimer: This is a PDF file of an unedited manuscript that has been accepted for publication. As a service to our customers we are providing this early version of the manuscript. The manuscript will undergo copyediting, typesetting, and review of the resulting proof before it is published in its final citable form. Please note that during the production process errors may be discovered which could affect the content, and all legal disclaimers that apply to the journal pertain.
} 
${ }^{4}$ Leonard Davis Institute of Health Economics, University of Pennsylvania, Philadelphia, PA, USA

${ }^{5}$ HealthCore, Inc, Wilmington, DE, USA

${ }^{6}$ Division of Research, Kaiser Permanente Northern California, Oakland, CA, USA

${ }^{7}$ Rutgers Biomedical and Health Sciences, Newark, New Jersey, USA

${ }^{8}$ Division of Infectious Diseases, Department of Medicine, Perelman School of Medicine, University of Pennsylvania, Philadelphia, PA, USA

\section{Abstract}

Background and Aims-Medications are a major cause of acute liver failure (ALF) in the US, but no population-based studies have evaluated the incidence of ALF from drug-induced liver injury. We aimed to determine the incidence and outcomes of drug-induced ALF in an integrated healthcare system that approximates a population-based cohort.

Methods-We performed a retrospective cohort study using data from the Kaiser Permanente Northern California (KPNC) healthcare system between January 1, 2004 and December 31, 2010. We included all KPNC members $\geq 18$ y old with $\geq 6$ months of membership and hospitalization for potential ALF. The primary outcome was drug-induced ALF (defined as coagulopathy and hepatic encephalopathy without underlying chronic liver disease), determined by hepatologists who reviewed medical records of all KPNC members with inpatient diagnostic and laboratory criteria suggesting potential ALF.

Results-Among 5,484,224 KPNC members between 2004 and 2010, 669 had inpatient diagnostic and laboratory criteria indicating potential ALF. After medical record review, 62 (9.3\%) were categorized as having definite or possible ALF, and $32(51.6 \%)$ had a drug-induced etiology (27 definite, 5 possible). Acetaminophen was implicated in 18 events (56.3\%), dietary/ herbal supplements in $6(18.8 \%)$, antimicrobials in $2(6.3 \%)$, and miscellaneous medications in 6 $(18.8 \%)$. One patient with acetaminophen-induced ALF died (5.6\%; .06 events/1,000,000 personyears) compared to 3 patients with non-acetaminophen induced ALF (21.4\%; .18/1,000,000 person-years). Overall, 6 patients (18.8\%) underwent liver transplantation, and 22 patients $(68.8 \%)$ were discharged without transplantation. The incidence rates of any definite drug-induced ALF and acetaminophen-induced ALF were 1.61 events/1,000,000 person-years (95\% confidence interval, 1.06-2.35) events and 1.02 events/1,000,000 person-years (95\% confidence interval, $0.59-1.63)$, respectively.

Conclusions-Drug-induced ALF is uncommon, but over-the-counter products and dietary/ herbal supplements are its most common causes.

\section{Keywords}

drug-induced liver injury; acetaminophen; acute liver failure

Acute liver failure is defined by the rapid onset of coagulopathy and hepatic encephalopathy in patients without underlying liver disease. Among patients hospitalized with acute liver failure at tertiary care centers, the transplant-free survival rate is less than $50 \%{ }^{1-3}$ In the United States, nearly $60 \%$ of reported cases of acute liver failure are related to drug-induced 
liver injury, with many "indeterminate" cases thought to have an underlying drug-induced etiology. ${ }^{1,3-5}$ However, these prior data have been derived only from tertiary care centers.

Despite widely publicized cases of drug-induced acute liver failure related to acetaminophen and other medications, ${ }^{4,6-10}$ there are no population-based studies specifically evaluating the incidence of acute liver failure arising from drug-induced liver injury. ${ }^{11}$ Published data primarily consist of case reports and case series from liver transplant and/or tertiary care centers ${ }^{2,3,12-17}$ or adverse event reports to regulatory agencies. ${ }^{5,18-20}$ As a result, the most common culprit medications, incidence, characteristics, and outcomes of drug-induced acute liver failure in real-world settings are unknown.

The objective of this study was to conduct an assessment of the incidence and outcomes of drug-induced acute liver failure within a large, integrated healthcare system that approximates a population-based cohort ("population-representative" cohort).

\section{METHODS}

\section{Study Design and Data Source}

We conducted a retrospective cohort study within Kaiser Permanente Northern California (KPNC) between January 1, 2004 and December 31, 2010. ${ }^{21} \mathrm{KPNC}$ is an integrated health care organization consisting of 21 medical centers and 160 outpatient medical offices that provide comprehensive inpatient and outpatient services to approximately 3.2 million Northern California residents. Its membership is racially/ethnically, geographically (urban, suburban, and semi-rural areas), and socioeconomically diverse and approximates the region's underlying population compared with census data; thus, studies in this setting closely approximate population-based research..$^{22,23}$ Data collected by KPNC include: demographic information; outpatient and hospital diagnoses; procedures; inpatient and outpatient laboratory results; emergency and referral services at non-Kaiser Permanente facilities; and dispensed medications, including dosage, instructions for use, and days' supply. Prescription drug benefits are utilized by more than $90 \%$ of members, and prior analyses have established the accuracy of pharmacy data. ${ }^{24}$ Mortality databases integrate death certificate data from the California Department of Health Services and Social Security Administration Death Master File. The study was approved by the KPNC and University of Pennsylvania Institutional Review Boards.

\section{Study Patients}

KPNC members aged 18 years or older with at least 6 months of membership were identified as having a hospitalization with potential acute liver failure if they had: 1) a hospital International Classification of Disease, Ninth Revision, Clinical Modification (ICD-9-CM) diagnosis suggestive of acute liver failure or drug-induced hepatitis (Supplementary Table 1$)^{25-27}$, and 2) an inpatient international normalized ratio (INR) $\geq 1.5$ (off warfarin) and peak total bilirubin $25.0 \mathrm{mg} / \mathrm{dL}$ within 182 days before or after the index date (defined below). Prior data suggested that these ICD-9-CM diagnoses are typically recorded for patients with severe liver injury. ${ }^{25-27}$ The peak total bilirubin cutoff for identifying potential acute liver failure cases for further abstraction was based on the very 
low likelihood of acute liver failure occurring in the absence of a peak bilirubin level $25.0 \mathrm{mg} / \mathrm{dL} .5,28$ The index date was defined as the date of hospital admission with potential acute liver failure.

Since by definition, patients with pre-existing liver disease or medication-related coagulopathy are not eligible to be classified as having acute liver failure, we excluded from further review patients with: 1) an ICD-9-CM diagnosis of a pre-existing liver disease (Supplementary Table 2) prior to the index date; 2) serologic or virologic evidence of hepatitis $\mathrm{B}, \mathrm{C}$, or D prior to the index date; 3 ) serologic evidence of acute hepatitis A or E in the 30 days before through 30 days after the index date; or 4) warfarin prescribed within 182 days before through 182 days after the index date.

\section{Confirmation of Drug-Induced Acute Liver Failure Events}

All KPNC members with potential acute liver failure had their hospital medical records abstracted by two trained data abstractors. For each patient, data collected from these medical records (reviewed for all data listed in Supplementary Table 3), in addition to the hospital discharge summary and gastroenterologist/hepatologist consultation notes, were independently reviewed by two transplant hepatologists (K.A.F. and D.G.), who classified acute liver failure as: 1) definite, 2) possible, or 3) no event. Disagreement resulted in review by a third hepatologist (K.R.R.) to adjudicate the event.

A definite diagnosis of acute liver failure, based on American Association for the Study of Liver Diseases criteria, ${ }^{29}$ was defined as: 1 ) the absence of pre-existing liver disease, 2) coagulopathy (INR $\geq 1.5$ ) in the absence of warfarin, and either 3a) hepatic encephalopathy (altered mentation due to liver dysfunction), or 3b) liver transplant with the stated indication of "acute liver failure/Status 1-the highest waitlist priority," without mention of hepatic encephalopathy in the available medical record (since United Network for Organ Sharing transplant policy requires hepatic encephalopathy as part of the classification of Status 1 criteria for acute liver failure $)^{30}$. A possible diagnosis was confirmed if a patient met criteria 1 and 2 (above) and had either: 1) altered mentation in the absence of a recorded diagnosis of hepatic encephalopathy but with encephalopathy treatment (e.g., lactulose, rifaximin) and no other explanatory central nervous system abnormality (e.g., acute intracranial ischemia), or 2) liver transplant during the index admission due to an acute etiology without specification of "acute liver failure/Status 1." Patients with documented chronic alcohol abuse based on medical record review and who had laboratory (e.g., thrombocytopenia), radiographic, and/or clinical evidence of chronic liver disease or portal hypertension were not classified as having acute liver failure. The final diagnosis of acute liver failure did not require a peak total bilirubin $25.0 \mathrm{mg} / \mathrm{dL}$ at the time of encephalopathy and coagulopathy.

\section{Etiology of acute liver failure}

The etiology of each acute liver failure event was ascertained by medical record review, and required the exclusion of other causes of acute liver failure, along with supporting clinical and laboratory information. Determination of the cause of acute liver failure and any implicated drugs or dietary/herbal supplements was based on consensus opinion among trained hepatologists with expertise in adjudicating drug-induced liver injury. There is no 
accepted international gold-standard for determining causality and classifying patients as having drug-induced liver injury. Although consensus opinion is widely used in the US for research purposes, it is not practical from a clinical perspective. Further, investigators from Europe and pharmaceutical companies are supportive of the RUCAM. ${ }^{31}$ We relied on consensus opinion in order for a patient to be classified as drug or supplement-induced acute liver failure, and required documentation of a potential etiologic drug or medication, in addition to exclusion of other potential etiologies based on laboratory or medical record review, including: 1) autoimmune hepatitis, based on consistent serologies and/or histology; ${ }^{32-34}$ 2) ischemic acute liver failure, based on acute elevations in liver aminotransferases in the context of a documented hypoperfusion event (e.g., septic, hypovolemic, or cardiogenic shock); 3) acute viral hepatitis confirmed by serologic or virologic tests (including hepatitis E virus and herpes simplex virus, when tested); 4) acute alcoholic hepatitis based on liver enzyme elevation pattern and clinical history given the presence of underlying fibrosis/chronic liver disease in such patients; and 5) vascular/ structural abnormalities (i.e. Budd-Chiari syndrome) based on available imaging reports. Acetaminophen was designated as the causative agent if there was documented excess ingestion of acetaminophen or acetaminophen-containing products and/or an elevated serum acetaminophen level (when available). A similar methodology for determination of a druginduced etiology for acute liver injury has been employed by the U.S. Drug-Induced Liver Injury Network. ${ }^{35,36}$ Cases were deemed indeterminate in the absence of any potential causative drug, medication, or any other identifiable etiology.

Although the Roussel Uclaf Causality Assessment Method (RUCAM) is an instrument that was developed to quantify the strength of the association between liver injury and an implicated medication, ${ }^{37,38}$ it was not used in this study because it: 1 ) has not been validated outside of clinical trials settings, 2) is unable to ascertain causality when two or more drugs may be implicated, and 3) is primarily applicable for idiosyncratic (non-acetaminophenbased) liver injury and cannot be used to identify dietary/herbal supplement-induced liver injury. 39

The time from onset of jaundice (defined as the first date of a serum total bilirubin $\geq 2.0$ $\mathrm{mg} / \mathrm{dL}^{2}$ ) to date of first clinical manifestation of hepatic encephalopathy was determined for each patient with drug-induced acute liver failure.

\section{Data Analysis}

Incidence rates of drug- and acetaminophen-induced acute liver failure were determined by dividing the number of KPNC members with confirmed events by the total person-years of follow-up. Person-years within the underlying KPNC population were estimated using the mid-year population for each year during the study period. ${ }^{40}$ Characteristics between patients with acetaminophen and non-acetaminophen-induced acute liver failure were compared using the Wilcoxon rank-sum test. Data were analyzed using Stata 13 (Stata Corporation, College Station, TX). 


\section{RESULTS}

Between January 1, 2004 and December 31, 2010, there were 5,484,224 unique members enrolled in KPNC, of whom 4,281,530 were aged 18 years or older. This corresponded to an average mid-year population over the age of 18 years of approximately 2.4 million (range: 2.35-2.45 million) members who contributed an estimated 16,731,307 person-years. Of these, 669 members had a hospitalization with potential acute liver failure, lacked preexisting liver disease based on recorded diagnoses or viral hepatitis serologic/virologic laboratory tests, and were not dispensed warfarin (Figure 1). Among these 669 members, 62 $(9.3 \%)$ were confirmed to have acute liver failure ( 45 definite, 17 possible) by medical record review. There was 93\% agreement in acute liver failure case arbitration between the two adjudicators, with a third hepatologist required to break disagreements in $7 \%$ of cases. Among the 607 classified as not having acute liver failure, 228 (37.6\%) were excluded due to pre-existing liver/biliary disease that was not detected by prior clinical, serologic, or virologic diagnosis (186 due to pre-existing alcoholic liver disease, 14 due to pre-existing autoimmune hepatitis, and 28 due to pre-existing cirrhosis), and 379 (62.4\%) had severe liver injury but no evidence of hepatic encephalopathy.

Of the 62 patients with confirmed acute liver failure, 32 (51.6\%) had a drug-induced etiology, with 27 (84.4\%) meeting criteria for definite acute liver failure (all with documented hepatic encephalopathy). Acetaminophen was implicated in 18 (56.3\%) events (17 definite), dietary/herbal supplements in 6 (18.8\%; four classified as definite), antimicrobials in two $(6.3 \%)$, and other medical products in $6(18.8 \%$; Table 1$)$. Among the 18 patients with acetaminophen-induced acute liver failure, $1(5.6 \% ; .06$ events/1,000,000 person-years) died, compared with 3/14 (21.4\%; .18/1,000,000 person-years) who died after developing non-acetaminophen induced acute liver failure.

Of the 32 patients with confirmed drug-induced liver failure, 12 (37.5\%) were transferred to a liver transplant center. When stratified by etiology of drug-induced acute liver failure (acetaminophen versus non-acetaminophen), the peak total bilirubin did not differ by transfer status in either group. However, the peak INR was significantly higher $(P=.01)$ in acetaminophen-induced acute liver failure patients who were transferred (median: 4.9, IQR: 3.9-5.7 versus 3.1, IQR: 2.1-3.8), but not among those with non-acetaminophen induced acute liver failure. Six (50.0\%) of the 12 transfers were transplanted, and $6(50.0 \%)$ were discharged alive without requiring a transplant. Of the remaining 20 patients who were not transferred, 4 (20.0\%) died in the hospital (3 of the 4 deaths categorized as "definite" acute liver failure), while $16(80.0 \%)$ were discharged alive without a transplant. Transplantationfree survival was more common among patients with drug-induced acute liver failure from acetaminophen (88.9\%) than from other agents (42.9\%; $P=.005$; Tables 1 and 2$)$.

In the 18 cases of acetaminophen-induced acute liver failure, four (22.2\%) had an intentional acetaminophen overdose alone, $3(16.7 \%)$ had unintentional overdoses in the setting of preceding alcohol abuse, $1(5.6 \%)$ had an intentional overdose with preceding alcohol abuse, and the remaining $10(55.6 \%)$ had an unintentional overdose without any evidence of alcohol abuse ( 2 of whom died). 
The incidence rate of any drug-induced acute liver failure (definite or possible) was 1.91 (95\% CI: 1.31-2.70) events per 1,000,000 person-years and the incidence rate of acetaminophen-induced acute liver failure (definite or possible) was 1.07 (95\% CI: 0.641.70) events per 1,000,000 person-years. Among only those with definite acute liver failure, the incidence rates of any drug-induced acute liver failure and acetaminophen-induced acute liver failure were 1.61 (95\% CI: 1.06-2.35) and 1.02 (95\% CI: 0.59-1.63) events per $1,000,000$ person-years, respectively.

Patients with acute liver failure due to acetaminophen were younger (median: 39 versus 63 years; $P=.008$ ), had higher peak levels of alanine aminotransferase (median: 4,588 versus $707 \mathrm{U} / \mathrm{L} ; \mathrm{p}<0.001$ ) and aspartate aminotransferase (median: 6,839 versus $1,044 \mathrm{U} / \mathrm{L}$; $\mathrm{p}<0.001$ ), had lower peak levels of total bilirubin ( 7.4 versus $20.6 \mathrm{mg} / \mathrm{dL} ; P=.01$; Table 2), and had shorter median times from jaundice to encephalopathy (median: 2 versus 8 days; $P<.001)$. The time from onset of jaundice to hepatic encephalopathy was longest in patients with drug-induced acute liver failure resulting from dietary/herbal supplements or antimicrobials (Table 2). Twenty-two (68.8\%) patients with drug-induced acute liver failure had time from jaundice to hepatic encephalopathy within 7 days ("hyperacute" acute liver failure $^{2}$ ).

In the 30 cases of non-drug-induced acute liver failure, the etiologies were: ischemia due to hypoperfusion (15/30); indeterminate etiology without any potential culprit drugs (6/30); malignancy (4/30); autoimmune hepatitis (2/30); and acute fatty liver of pregnancy, acute hepatitis $\mathrm{B}$, and Wilson's disease (1/30 for each).

\section{CONCLUSIONS}

Nearly $50 \%$ of Americans take one or more prescription medications every month, ${ }^{41} 15 \%$ consume over-the-counter analgesics daily, ${ }^{42}$ and almost $20 \%$ use dietary or herbal supplements regularly. ${ }^{43}$ Untoward effects of medication and supplement use are an important public health concern, particularly as the population ages and medication usage increases. In this study, we report an incidence of definite drug-induced acute liver failure of 1.61 per million person-years in an integrated healthcare system that approximates a population-based cohort, with the overwhelming majority related to over-the-counter medications or products. These data provide a better understanding of the true populationrepresentative incidence of drug-induced acute liver failure, the most severe form of druginduced hepatotoxicity, and its associated morbidity and mortality in an integrated health care organization.

An important observation was the role of over-the-counter medications and supplements. Dietary/herbal supplements were implicated in $18.8 \%$ of drug-induced acute liver failure cases. Combined with acetaminophen, $75 \%$ of drug-induced acute liver failure cases in this study were attributed to over-the-counter products, with over one-half of the acetaminophen overdoses being unintentional. Such results stand in stark contrast to data from countries where acetaminophen is infrequently combined with other painkillers, and is not a cause of acute liver failure (e.g., no cases of acetaminophen-induced acute liver failure reported in a six and a half-year period in Argentina ${ }^{44}$ ). Stricter regulation of co-formulation of 
acetaminophen with other painkillers or limiting the number of acetaminophen tablets available for over-the-counter purchase might help to decrease the number of acetaminophen-related acute liver failure cases. Additionally, while acetaminophen has proven health benefits, most dietary/herbal supplements do not have demonstrated efficacy for the treatment or prevention of particular diseases, and these products currently do not undergo pre-marketing safety assessments by the Food and Drug Administration. ${ }^{45}$ These data suggest that standard prescription medications are a rare source of acute liver failure; although there is an ongoing need for continued vigilant reporting and prospective studies of hepatotoxicity and acute liver failure among users of these products, consideration should also be given to additional pre-marketing regulatory oversight of dietary supplements and herbal products, as well as additional post-marketing efforts to identify drug-, herbal-, and dietary supplement-induced liver injury. Furthermore, given that over one-half of cases of acetaminophen-induced acute liver failure were from unintentional overdoses, consideration should be given to laws that limit the size of packs of acetaminophen, and/or require acetaminophen packages to use blister packs. Such legislation enacted in the United Kingdom in 1988 resulted in a significant reduction in cases of acute liver failure from acetaminophen. ${ }^{46}$

Although prescription drugs were a rare cause of drug-induced acute liver failure in this cohort, the medications that were implicated were consistent with previously published reports. Isoniazid and amoxicillin/clavulanate have been implicated as two of the most common antimicrobial agents that lead to drug-induced liver injury and acute liver failure. ${ }^{47-51}$ Similarly, chemotherapeutic and immunosuppressant agents ${ }^{52-54}$, as well as antiretroviral drugs ${ }^{55-57}$, have also been reported as causes of severe drug-induced liver injury and acute liver failure. In clinical practice, the risks and benefits of prescribing such medications should be made on a case-by-case basis and in the context of the exceedingly rare risk of drug-induced acute liver failure.

These data allow for a better understanding of the incidence, etiology, and outcomes of acute liver failure in a community-based population. Approximately $25 \%$ of Northern California residents have KPNC health insurance, thus these data capture a large proportion of the adult population in Northern California. Based on data derived from the California Health Interview Study, the KPNC membership is similar to the broader Northern California population with regards to socio-demographic and health characteristics. ${ }^{24}$

The transplant-free survival of the patients with acetaminophen- versus non-acetaminopheninduced acute liver failure was not significantly different. However, when put in the context of previously reported data, the transplant-free survival in the non-acetaminophen group was similar to that previously reported by the US Acute Liver Failure Study Group, the transplant-free survival of nearly $90 \%$ in acetaminophen-induced acute liver failure was substantially higher in the current study. ${ }^{3,9}$ While a potential explanation is the nature of these data, such that nearly two-thirds of our subjects with drug-induced acute liver failure never received care at a tertiary care center despite being critically ill, this alone cannot account for the higher survival given that patients with acetaminophen-induced acute liver failure who were not transferred to a tertiary care center had INR and total bilirubin values comparable to those previously reported by the US Acute Liver Failure Study Group. ${ }^{1,3}$ Yet 
the current patients had substantially higher transplant-free survival than those previously reported. ${ }^{1,3,9,58}$ While the degree of encephalopathy in non-transferred patients may have been less severe than those that were transferred, which cannot be evaluated retrospectively, all of these patients nevertheless met criteria for possible or definite acute liver failure. Although it cannot be directly proven in the context of this study, one hypothesis for the higher transplant-free survival rate in these patients may, in part, be related to being cared for in a coordinated health care network that links patients, doctors, and hospitals together to optimize patient care. Nevertheless, the risk of mortality from APAP-related ALF and nonAPAP related ALF was small, at less than 1 event per 1,000,000 person-years, respectively.

The higher transplant-free survival may be attributable to other factors, including: 1) improved management of acute liver failure and critically ill patients over time; 2) earlier recognition and intervention of acetaminophen overdose with available therapy (i.e., nacetylcysteine); or 3) unmeasured confounders. Finally, the results confirm that, even in a community-based setting, the patterns of liver test abnormalities and bilirubin elevations in the acetaminophen- and non-acetaminophen-induced acute liver failure cases are consistent with prior reports. $3,4,8,9,12,17,42,58,59$

Our study has several limitations. First, we used ICD-9-CM diagnoses to exclude patients with non-viral chronic liver disease, and there is the potential that some patients might have been misclassified as having underlying liver disease. Second, we restricted medical record review to patients with a peak total bilirubin $25.0 \mathrm{mg} / \mathrm{dL}$, given the low likelihood of acute liver failure developing without a peak total bilirubin reaching this value. There is the potential that we might have missed a very small number of patients with acute liver failure who never obtained this total bilirubin level, in particular those with acetaminophen-induced acute liver failure ${ }^{5,28}$ However, even if the total incidence of drug-induced acute liver failure was underestimated by as much as $25 \%$, the incidence of drug-induced acute liver failure would only increase to 2.57 cases per $1,000,000$ person-years. Third, the data were reviewed retrospectively, and certain laboratory tests listed in Supplementary Table 3 (e.g., hepatitis E serologies) were missing for some patients. Nevertheless, determination of the etiology of acute liver failure was based on independent adjudication by at least two hepatologists. Additionally, there were no events deemed to be drug-induced where a competing potential etiology was considered, yet not diagnosed, because of missing laboratory testing (e.g., a case where the diagnosis was deemed drug-induced, but the patient lacked test results for other diagnoses, such as quantitative immunoglobulins for autoimmune hepatitis). Fourth, alcohol history was based on retrospective review of the medical record, which may have led to misclassification of chronic alcohol use. However, this limitation may also potentially exist in prospectively collected data if patients do not report alcohol use, and any misclassification would likely lead to under-recognition of chronic alcohol use, and thus over-estimation of true drug-induced acute liver failure in the absence of chronic alcohol use. Fifth, the exact preparation, and purchase location of the dietary/herbal supplements that led to acute liver failure were not known.

Although this study was conducted in Northern California, we believe these results are generalizable to broader populations. Previous work within the KPNC integrated health care network, which includes greater than 1 of every 100 people in the United States, has 
demonstrated that the KPNC population closely reflects the broader population in Northern California. ${ }^{22,23}$ The estimates of the number of acute liver failure events in this cohort are also consistent with data from two other distinct cohorts of patients, which further suggests the generalizability of these data. First, according to Organ Procurement and Transplantation Network data, approximately 50 adults are transplanted each year for drug-induced acute liver failure, with an additional 40 for unknown etiologies. Given that the 2.4 million KPNC members in this analysis represent approximately $1 \%$ of the US adult population, our observation of six transplants for drug-induced acute liver failure within seven years is consistent with the reported annual average number of transplants for drug-induced acute liver failure. ${ }^{60}$ Second, our estimates of the proportion of acute liver failure cases due to drugs are consistent with results of the multi-center US Acute Liver Failure Study Group. ${ }^{3,5}$ Furthermore, recent data from the Drug-Induced Liver Injury Network also suggest that an increasing proportion of cases of drug-induced liver injury in the U.S. are arising from dietary/herbal supplements. ${ }^{17}$ These lines of evidence further support the accuracy of our methods for identifying acute liver failure events.

The specific drugs or supplements implicated as causing acute liver failure in this study differed from a previous report of liver transplant recipients with acute liver failure due to drug-induced liver injury published in $2004 .{ }^{61}$ This difference is likely due to the different time periods of the two studies (1990-2002 versus 2004-2010), with the first study including medications that are no longer available on the market (specifically halothane and troglitazone), as well as the spectrum of diseases in the two cohorts (transplant recipients versus patients with acute liver failure, regardless of transplant status). ${ }^{61}$

In conclusion, we found that the incidence of drug-induced acute liver failure was 1.61 per million person-years in adults in a population-representative cohort, and acetaminophen and dietary/herbal supplements were implicated in three-quarters of cases. These data highlight the rarity of this complication, and provide estimates of the true risk of acute liver failure resulting from medications, herbals, and/or dietary supplements. Furthermore, such events are rarely due to prescription medications. While continued vigilance for acute liver injury from prescription medications remains important, these data suggest that closer attention to the hepatotoxicity of over-the-counter medications, particularly dietary and herbal supplements, is needed.

\section{Supplementary Material}

Refer to Web version on PubMed Central for supplementary material.

\section{Acknowledgments}

\section{Grant Support Information}

1. Vincent Lo Re III: Agency for Healthcare Research and Quality R01 HS018372

2. David Goldberg: NIH K08 DK098272

3. Kimberly Forde: K23 DK090209

4. James Lewis: K24 DK078228 


\section{Abbreviations}
ALF
Acute liver failure
KPNC
Kaiser Permanente Northern California
ICD-9-CM
International Classification of Disease, Ninth Revision, Clinical Modification
RUCAM Roussel Uclaf Causality Assessment Method

\section{References}

1. Lee WM. Acute liver failure in the United States. Semin Liver Dis. 2003; 23(3):217-226. [PubMed: 14523675]

2. O’Grady JG, Schalm SW, Williams R. Acute liver failure: redefining the syndromes. Lancet. 1993; 342(8866):273-275. [PubMed: 8101303]

3. Ostapowicz G, Fontana RJ, Schiodt FV, et al. Results of a prospective study of acute liver failure at 17 tertiary care centers in the United States. Ann Intern Med. 2002; 137(12):947-954. [PubMed: 12484709]

4. Lee WM. Drug-induced hepatotoxicity. N Engl J Med. 2003; 349(5):474-485. [PubMed: 12890847]

5. Reuben A, Koch DG, Lee WM. Acute Liver Failure Study G. Drug-induced acute liver failure: results of a U.S. multicenter, prospective study. Hepatology. 2010; 52(6):2065-2076. [PubMed: 20949552]

6. Chalasani N, Vuppalanchi R, Navarro V, et al. Acute liver injury due to flavocoxid (Limbrel), a medical food for osteoarthritis: a case series. Ann Intern Med. 2012; 156(12):857-860. W297-300. [PubMed: 22711078]

7. Forman LM, Simmons DA, Diamond RH. Hepatic failure in a patient taking rosiglitazone. Ann Intern Med. 2000; 132(2):118-121. [PubMed: 10644272]

8. Laine L, Goldkind L, Curtis SP, Connors LG, Yanqiong Z, Cannon CP. How common is diclofenacassociated liver injury? Analysis of 17,289 arthritis patients in a long-term prospective clinical trial. Am J Gastroenterol. 2009; 104(2):356-362. [PubMed: 19174782]

9. Larson AM, Polson J, Fontana RJ, et al. Acetaminophen-induced acute liver failure: results of a United States multicenter, prospective study. Hepatology. 2005; 42(6):1364-1372. [PubMed: 16317692]

10. Vuppalanchi R, Hayashi PH, Chalasani N, et al. Duloxetine hepatotoxicity: a case-series from the drug-induced liver injury network. Aliment Pharmacol Ther. 2010; 32(9):1174-1183. [PubMed: 20815829]

11. Bower WA, Johns M, Margolis HS, Williams IT, Bell BP. Population-based surveillance for acute liver failure. Am J Gastroenterol. 2007; 102(11):2459-2463. [PubMed: 17608778]

12. Escorsell A, Mas A, de la Mata M. Spanish Group for the Study of Acute Liver F. Acute liver failure in Spain: analysis of 267 cases. Liver Transpl. 2007; 13(10):1389-1395. [PubMed: 17370334]

13. Hadem J, Tacke F, Bruns T, et al. Etiologies and outcomes of acute liver failure in Germany. Clin Gastroenterol Hepatol. 2012; 10(6):664-669. e662. [PubMed: 22373724]

14. Schiodt FV, Atillasoy E, Shakil AO, et al. Etiology and outcome for 295 patients with acute liver failure in the United States. Liver Transpl Surg. 1999; 5(1):29-34. [PubMed: 9873089]

15. Andrade RJ, Lucena MI, Fernandez MC, et al. Drug-induced liver injury: An analysis of 461 incidences submitted to the Spanish Registry over a 10-year period. Gastroenterology. 2005; 129(2):512-521. [PubMed: 16083708]

16. Bjornsson E, Olsson R. Outcome and prognostic markers in severe drug-induced liver disease. Hepatology. 2005; 42(2):481-489. [PubMed: 16025496] 
17. Navarro VJ, Barnhart H, Bonkovsky HL, et al. Liver injury from herbals and dietary supplements in the U.S. Drug-Induced Liver Injury Network. Hepatology. 2014; 60(4):1399-1408. [PubMed: 25043597]

18. [Accessed January 13, 2015] Liver Toxicity Knowledge Base (LTKB). http://www.fda.gov/ ScienceResearch/BioinformaticsTools/LiverToxicityKnowledgeBase/ucm2024036.htm

19. Hoofnagle JH, Serrano J, Knoben JE, Navarro VJ. LiverTox: a website on drug-induced liver injury. Hepatology. 2013; 57(3):873-874. [PubMed: 23456678]

20. Senior JR. Drug hepatotoxicity from a regulatory perspective. Clin Liver Dis. 2007; 11(3):507524. vi. [PubMed: 17723917]

21. Friedman, G.; Habel, L.; Boles, M.; McFarland, B. Kaiser Permanente Medical Care Program: Division of Research, Northern California, and Center for Health Research, Northwest Division. In: Strom, BL., editor. Pharmacoepidemiology. 3. West Sussex: John Wiley \& Sons, Ltd; 2000. p. 263-283.

22. Koebnick C, Langer-Gould AM, Gould MK, et al. Sociodemographic characteristics of members of a large, integrated health care system: comparison with US Census Bureau data. Perm J. 2012; 16(3):37-41. [PubMed: 23012597]

23. NPG. [Accessed 12/23/2013] How Does the Adult Kaiser Permanente Membership in Northern California Compare with the Larger Community?. 2006. http://www.dor.kaiser.org/external/ uploadedFiles/content/research/mhs/_2011_Revised_Site/Documents_Special_Reports/ comparison_kaiser_vs_nonKaiser_adults_kpnc(1).pdf

24. Schatz M, Zeiger RS, Vollmer WM, et al. Validation of a beta-agonist long-term asthma control scale derived from computerized pharmacy data. J Allergy Clin Immunol. 2006; 117(5):995-1000. [PubMed: 16675324]

25. Jinjuvadia K, Kwan W, Fontana RJ. Searching for a needle in a haystack: use of ICD-9-CM codes in drug-induced liver injury. Am J Gastroenterol. 2007; 102(11):2437-2443. [PubMed: 17662100]

26. Myers RP, Leung Y, Shaheen AA, Li B. Validation of ICD-9-CM/ICD-10 coding algorithms for the identification of patients with acetaminophen overdose and hepatotoxicity using administrative data. BMC Health Serv Res. 2007; 7:159. [PubMed: 17910762]

27. Lo Re V 3rd, Haynes K, Goldberg D, et al. Validity of diagnostic codes to identify cases of severe acute liver injury in the US Food and Drug Administration's Mini-Sentinel Distributed Database. Pharmacoepidemiol Drug Saf. 2013; 22(8):861-872. [PubMed: 23801638]

28. Robles-Diaz M, Lucena MI, Kaplowitz N, et al. Use of Hy's law and a new composite algorithm to predict acute liver failure in patients with drug-induced liver injury. Gastroenterology. 2014; 147(1):109-118. e105. [PubMed: 24704526]

29. Polson J, Lee WM. AASLD position paper: the management of acute liver failure. Hepatology. 2005; 41(5):1179-1197. [PubMed: 15841455]

30. [Accessed October 2, 2014] OPTN/UNOS Policy 3.7.B: Required Expedited Modifications of Waiting Time. http://optn.transplant.hrsa.gov/ContentDocuments/OPTN_Policies.pdf

31. Aithal GP, Watkins PB, Andrade RJ, et al. Case definition and phenotype standardization in druginduced liver injury. Clin Pharmacol Ther. 2011; 89(6):806-815. [PubMed: 21544079]

32. Alvarez F, Berg PA, Bianchi FB, et al. International Autoimmune Hepatitis Group Report: review of criteria for diagnosis of autoimmune hepatitis. J Hepatol. 1999; 31(5):929-938. [PubMed: 10580593]

33. Manns MP, Czaja AJ, Gorham JD, et al. Diagnosis and management of autoimmune hepatitis. Hepatology. 2010; 51(6):2193-2213. [PubMed: 20513004]

34. Vergani D, Alvarez F, Bianchi FB, et al. Liver autoimmune serology: a consensus statement from the committee for autoimmune serology of the International Autoimmune Hepatitis Group. J Hepatol. 2004; 41(4):677-683. [PubMed: 15464251]

35. Fontana RJ, Watkins PB, Bonkovsky HL, et al. Drug-Induced Liver Injury Network (DILIN) prospective study: rationale, design and conduct. Drug Saf. 2009; 32(1):55-68. [PubMed: 19132805]

36. Rockey DC, Seeff LB, Rochon J, et al. Causality assessment in drug-induced liver injury using a structured expert opinion process: comparison to the Roussel-Uclaf causality assessment method. Hepatology. 2010; 51(6):2117-2126. [PubMed: 20512999] 
37. Danan G, Benichou C. Causality assessment of adverse reactions to drugs--I. A novel method based on the conclusions of international consensus meetings: application to drug-induced liver injuries. J Clin Epidemiol. 1993; 46(11):1323-1330. [PubMed: 8229110]

38. Benichou C, Danan G, Flahault A. Causality assessment of adverse reactions to drugs--II. An original model for validation of drug causality assessment methods: case reports with positive rechallenge. J Clin Epidemiol. 1993; 46(11):1331-1336. [PubMed: 8229111]

39. Garcia-Cortes M, Stephens C, Lucena MI, Fernandez-Castaner A, Andrade RJ. Spanish Group for the Study of Drug-Induced Liver D. Causality assessment methods in drug induced liver injury: strengths and weaknesses. J Hepatol. 2011; 55(3):683-691. [PubMed: 21349301]

40. SP. Demography: Measuring and modeling population processes. Blackwell Publishers; 2001.

41. Centers for Disease Control and Prevention. [Accessed November 15, 2013] Therapeutic Drug Use. http://www.cdc.gov/nchs/fastats/drugs.htm

42. Wilcox CM, Cryer B, Triadafilopoulos G. Patterns of use and public perception of over-thecounter pain relievers: focus on nonsteroidal antiinflammatory drugs. J Rheumatol. 2005; 32(11): 2218-2224. [PubMed: 16265706]

43. Bailey RL, Gahche JJ, Lentino CV, et al. Dietary supplement use in the United States, 2003-2006. J Nutr. 2011; 141(2):261-266. [PubMed: 21178089]

44. Mendizabal M, Marciano S, Videla MG, et al. Changing etiologies and outcomes of acute liver failure: Perspectives from 6 transplant centers in Argentina. Liver Transpl. 2014; 20(4):483-489. [PubMed: 24425668]

45. U.S. Food and Drug Administration. [Accessed November 18, 2013] Dietary Supplements. http:// www.fda.gov/food/dietarysupplements/

46. Hawton K, Simkin S, Deeks J, et al. UK legislation on analgesic packs: before and after study of long term effect on poisonings. BMJ. 2004; 329(7474):1076. [PubMed: 15516343]

47. Bjornsson ES, Bergmann OM, Bjornsson HK, Kvaran RB, Olafsson S. Incidence, presentation, and outcomes in patients with drug-induced liver injury in the general population of Iceland. Gastroenterology. 2013; 144(7):1419-1425. 1425 e1411-1413. quiz e1419-1420. [PubMed: 23419359]

48. Thiim M, Friedman LS. Hepatotoxicity of antibiotics and antifungals. Clin Liver Dis. 2003; 7(2): 381-399. vi-vii. [PubMed: 12879990]

49. Nash KL, Yeung TM, Lehner PJ, Gibbs P, Griffiths WJ. Orthotopic liver transplantation for subacute hepatic failure following partial treatment of isoniazid-resistant tuberculosis. Transpl Infect Dis. 2008; 10(4):272-275. [PubMed: 17868274]

50. Hasagawa T, Reyes J, Nour B, et al. Successful liver transplantation for isoniazid-induced hepatic failure--a case report. Transplantation. 1994; 57(8):1274-1277. [PubMed: 8178358]

51. Farrell FJ, Keeffe EB, Man KM, Imperial JC, Esquivel CO. Treatment of hepatic failure secondary to isoniazid hepatitis with liver transplantation. Dig Dis Sci. 1994; 39(10):2255-2259. [PubMed: 7924752]

52. Bilasy SE, Essawy SS, Mandour MF, Ali EA, Zaitone SA. Myelosuppressive and hepatotoxic potential of leflunomide and methotrexate combination in a rat model of rheumatoid arthritis. Pharmacol Rep. 2015; 67(1):102-114. [PubMed: 25560583]

53. Kong JH, Yoo SH, Lee KE, et al. Early imatinib-mesylate-induced hepatotoxicity in chronic myelogenous leukaemia. Acta Haematol. 2007; 118(4):205-208. [PubMed: 18030002]

54. Floyd J, Mirza I, Sachs B, Perry MC. Hepatotoxicity of chemotherapy. Semin Oncol. 2006; 33(1): 50-67. [PubMed: 16473644]

55. Tseng YT, Yang CJ, Chang SY, et al. Incidence and risk factors of skin rashes and hepatotoxicity in HIV-infected patients receiving nevirapine-containing combination antiretroviral therapy in Taiwan. Int J Infect Dis. 2014; 29:12-17. [PubMed: 25312984]

56. Mulu W, Gidey B, Chernet A, Alem G, Abera B. Hepatotoxicity and associated risk factors in HIV-infected patients receiving antiretroviral therapy at Felege Hiwot Referral Hospital, Bahirdar, Ethiopia. Ethiop J Health Sci. 2013; 23(3):217-226. [PubMed: 24307821]

57. Vogel M, Rockstroh JK. Hepatotoxicity and liver disease in the context of HIV therapy. Curr Opin HIV AIDS. 2007; 2(4):306-313. [PubMed: 19372904] 
58. Lee WM, Squires RH Jr, Nyberg SL, Doo E, Hoofnagle JH. Acute liver failure: Summary of a workshop. Hepatology. 2008; 47(4):1401-1415. [PubMed: 18318440]

59. Navarro VJ, Senior JR. Drug-related hepatotoxicifty. N Engl J Med. 2006; 354(7):731-739. [PubMed: 16481640]

60. According to OPTN/UNOS data as of January 10.

61. Russo MW, Galanko JA, Shrestha R, Fried MW, Watkins P. Liver transplantation for acute liver failure from drug induced liver injury in the United States. Liver Transpl. 2004; 10(8):1018-1023. [PubMed: 15390328] 


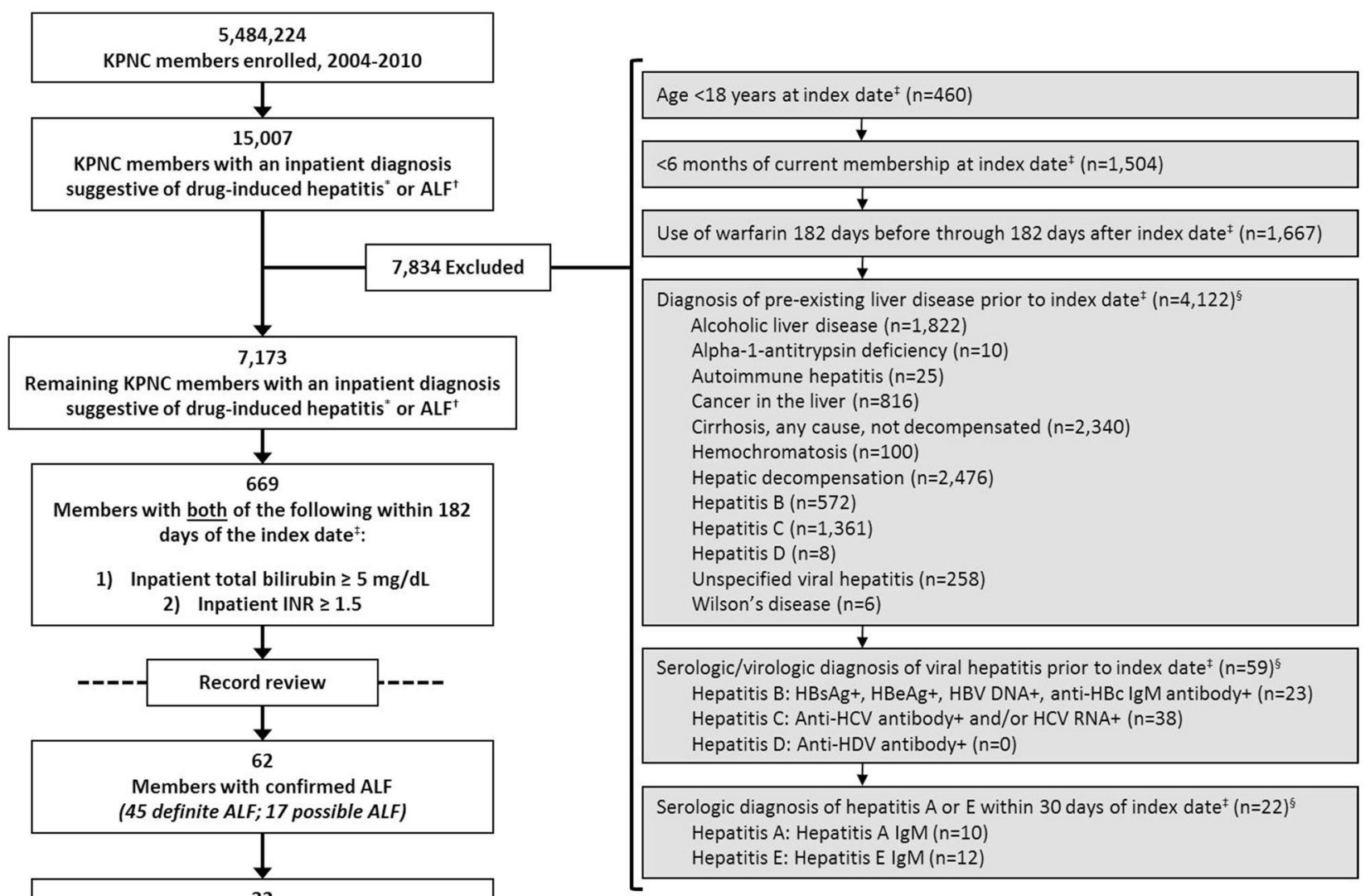

Members with confirmed drug-induced ALF

(27 definite ALF; 5 possible ALF)

Figure 1. Flow diagram of study patients

KPNC=Kaiser Permanente Northern California; ALF=acute liver failure; INR=international normalized ratio; DNA=deoxyribonucleic acid; RNA=ribonucleic acid

*ICD-9 codes 573.3 or 573.8

$\dagger$ ICD-9 codes 570, 572.2, 572.4, 572.8, V42.7

FIndex date defined as the admission date of the hospitalization associated with the druginduced hepatitis or ALF diagnosis code

§Patients may have had more than one exclusionary diagnosis recorded 


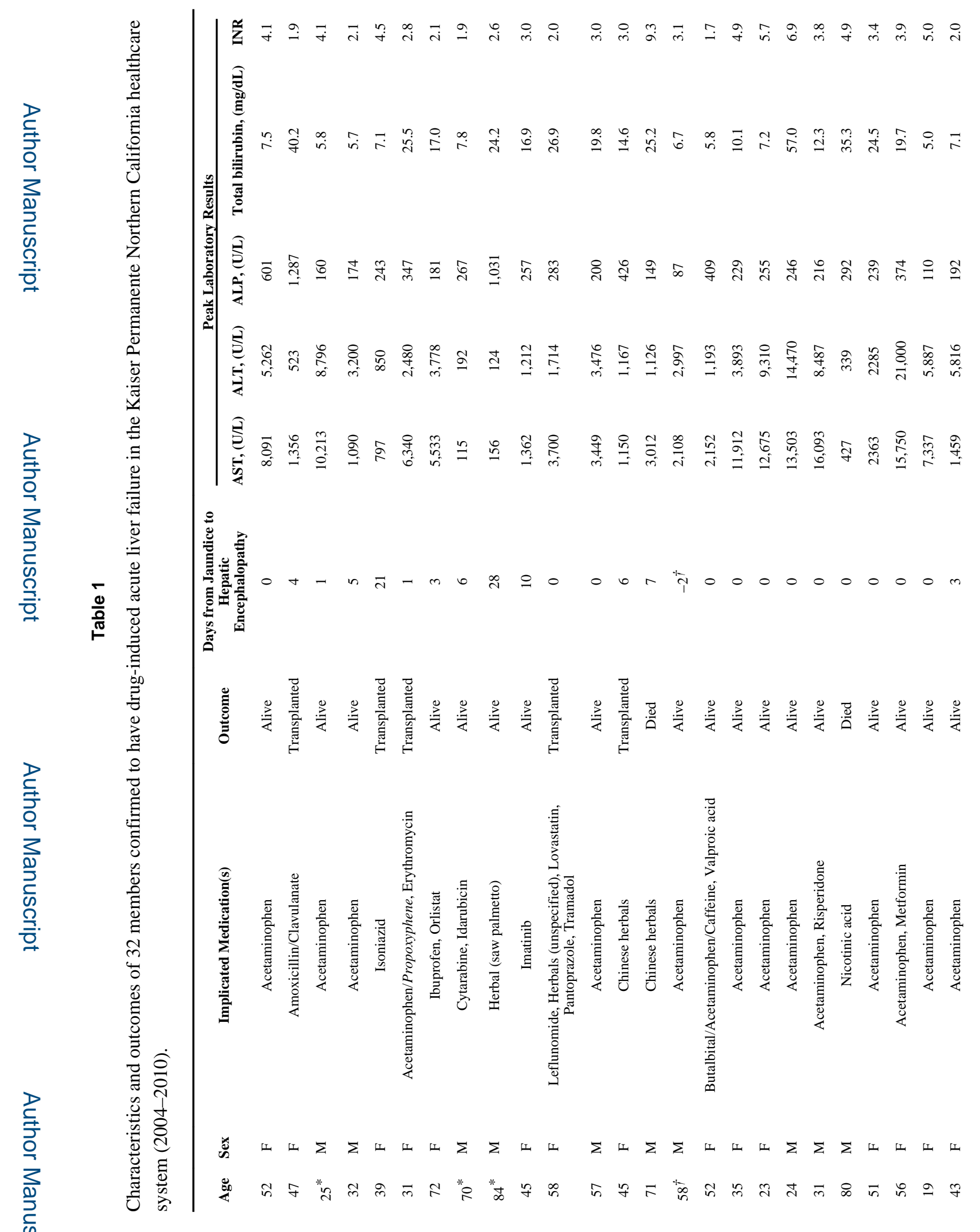

Gastroenterology. Author manuscript; available in PMC 2016 June 01. 




Gastroenterology. Author manuscript; available in PMC 2016 June 01. 


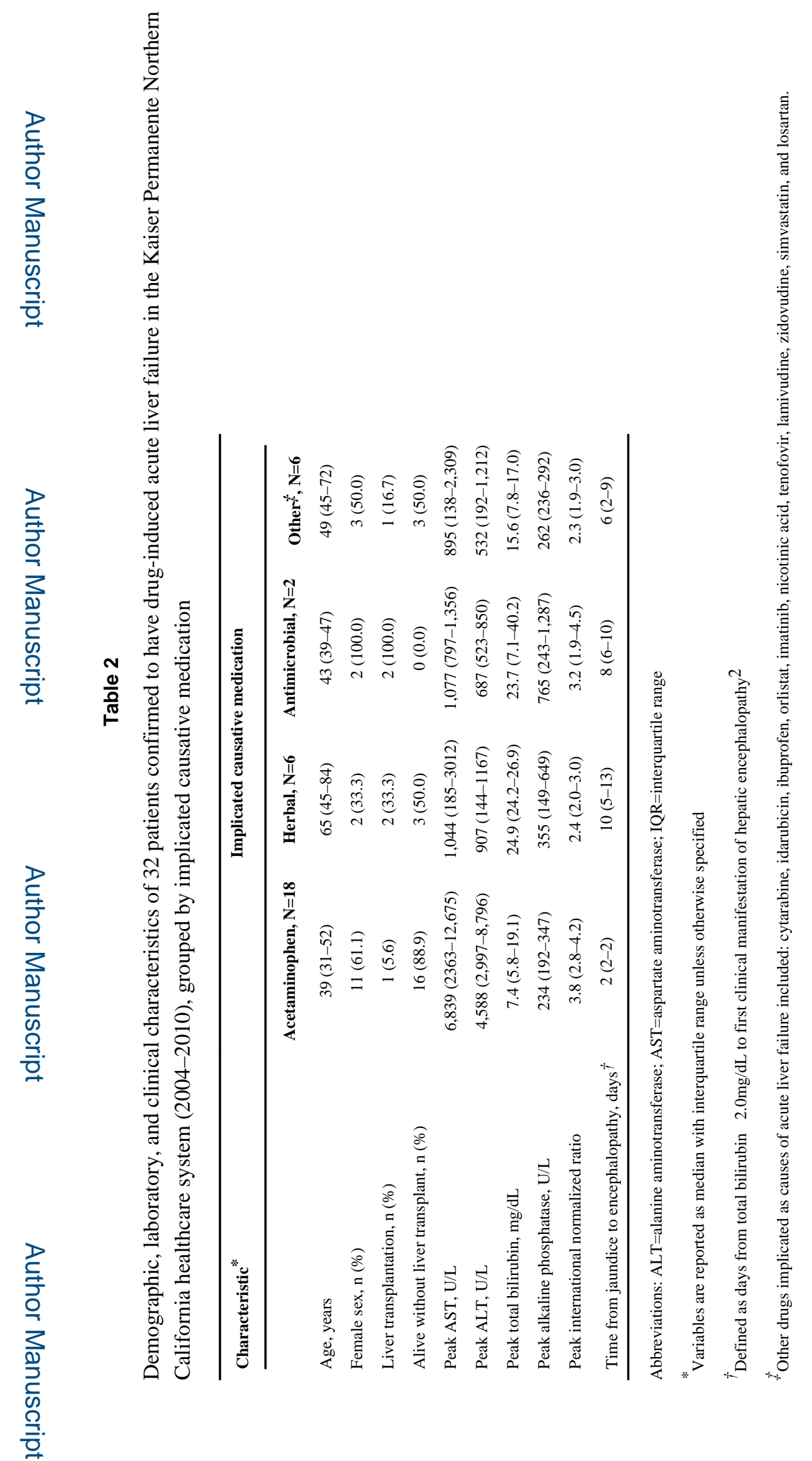

Gastroenterology. Author manuscript; available in PMC 2016 June 01. 